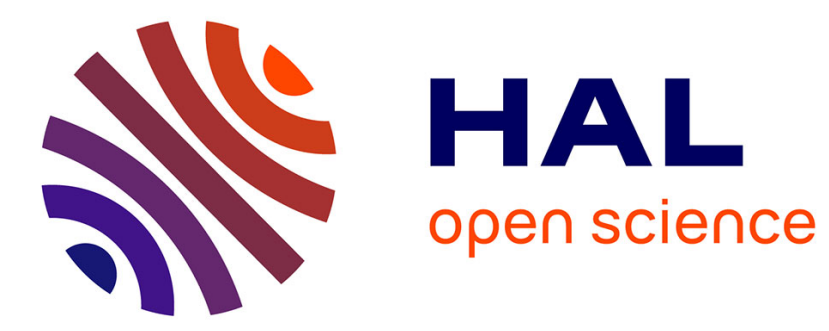

\title{
Indecisiveness aversion and preference for commitment
} Eric Danan, Ani Guerdjikova, Alexander Zimper

\section{To cite this version:}

Eric Danan, Ani Guerdjikova, Alexander Zimper. Indecisiveness aversion and preference for commitment. Theory and Decision, 2012, 72 (1), pp.1-13. 10.1007/s11238-011-9254-7 . hal-00872238

\section{HAL Id: hal-00872238 \\ https://hal.science/hal-00872238}

Submitted on 11 Oct 2013

HAL is a multi-disciplinary open access archive for the deposit and dissemination of scientific research documents, whether they are published or not. The documents may come from teaching and research institutions in France or abroad, or from public or private research centers.
L'archive ouverte pluridisciplinaire HAL, est destinée au dépôt et à la diffusion de documents scientifiques de niveau recherche, publiés ou non, émanant des établissements d'enseignement et de recherche français ou étrangers, des laboratoires publics ou privés. 


\title{
Indecisiveness aversion and preference for commitment*
}

\author{
Eric Danan ${ }^{\dagger} \quad$ Ani Guerdjikova ${ }^{\ddagger} \quad$ Alexander Zimper $^{\S}$
}

March 6, 2011

\begin{abstract}
We present an axiomatic model of preferences over menus that is motivated by three assumptions. First, the decision maker is uncertain ex ante (i.e. at the time of choosing a menu) about her ex post (i.e. at the time of choosing an option within her chosen menu) preferences over options, and she anticipates that this subjective uncertainty will not resolve before the ex post stage. Second, she is averse to ex post indecisiveness (i.e. to having to choose between options that she cannot rank with certainty). Third, when evaluating a menu she discards options that are dominated (i.e. inferior to another option whatever her ex post preferences may be) and restricts attention to the undominated ones. Under these assumptions, the decision maker has a preference for commitment in the sense of preferring menus with fewer undominated alternatives. We derive a representation in which the decision maker's uncertainty about her ex post preferences is captured by means of a subjective state space, which in turn determines which options are undominated in a given menu, and in which the decision maker fears, whenever indecisive, to choose an option that will turn out to be the worst (undominated) one according to the realization of her ex post preferences.
\end{abstract}

Keywords. Opportunity sets, subjective uncertainty, indecisiveness, dominance. JEL Classification. D81.

\footnotetext{
${ }^{*}$ We thank Klaus Nehring, Prasanta Pattanaik, Wolfgang Pesendorfer, Jean-Christophe Vergnaud, two anonymous referees, as well as participants of FUR XIII in Barcelona and the First Transatlantic Theory Workshop in Paris for helpful comments and suggestions. Alexander Zimper gratefully acknowledges financial support from ERSA (Economic Research Southern Africa).

†Université de Cergy-Pontoise, THEMA, 33 boulevard du Port, 95000 Cergy-Pontoise, France. E-mail: eric.danan@u-cergy.fr.

${ }^{\ddagger}$ Université de Cergy-Pontoise, THEMA, 33 boulevard du Port, 95000 Cergy-Pontoise, France. E-mail: ani.guerdjikova@u-cergy.fr.

${ }^{\S}$ School of Economic and Business Sciences, University of the Witwatersrand, Johannesburg, South Africa. E-mail: alexander.zimper@wits.ac.za.
} 


\section{Introduction}

Consider a two-stage decision situation. In the first stage, the decision maker has to choose a menu (or opportunity set). In the second stage, she has to choose an option from this menu. We refer to these two stages as the ex ante and ex post stage, respectively. We assume that the decision maker is uncertain ex ante about her ex post preferences over options. Standard models in the literature on opportunity sets use this assumption in order to motivate a desire for flexibility (Kreps, 1979; Nehring, 1999; Dekel, Lipman, and Rustichini, 2001; Dekel, Lipman, Rustichini, and Sarver, 2007a; Ozdenoren, 2002; Epstein, Marinacci, and Seo, 2007). According to these models, larger menus can never be worse than smaller ones when a decision maker expects to learn her ex post preferences before actually having to choose an option. In contrast to these approaches, we consider a decision maker who anticipates that her uncertainty about her ex post preferences will only resolve after she will have chosen an option. Such a decision maker will find herself at the ex post stage, at least for some menus, in a situation of indecisiveness, i.e. of having to choose an option without being certain which option she prefers. We assume that the decision maker is averse to such situations of indecisiveness and, therefore, prefers smaller menus to larger ones, to the extent that smaller menus enable her to avoid these situations.

As an illustrative example, consider Bethy, who is a manager of a small division in a large company. She is faced with the problem of assigning the execution of a project to one of the employees. Right now she can only choose among the employees in her division, whom she knows well and has previously observed in similar projects. She is rather certain that Alan would be the best person to entrust with the project. However, just before Bethy can make the decision, the CEO of the company contacts her and suggests that she now has the possibility to pick an employee not just from her own division, but from the entire company staff. Bethy has only limited knowledge of the staff outside her division. In particular, she knows that Bob and Chris are well suited to execute the project, but she finds these two candidates hard to compare: e.g., Bob would be excellent on the financial side of the project, but Chris would do better than Bob when it comes to marketing. Bethy knows that these two dimensions are important for the success of the project, but the current situation makes it difficult to foresee which one will be the most important. She is faced with a hard choice: she has to make an important decision (for the company, for her career and that of the person who will be in charge of the project), and take full responsibility for this decision in front of the CEO, without being able to confidently go for either one of the possible options. In fact, she would have much preferred sticking to her division, which would have avoided her this situation of indecisiveness altogether. Thus she would be willing to forego candidates that are potentially better than Alan (in fact, she may even be sure that, e.g., Chris is superior 
to Alan in all regards) in order to avoid the pain of having to choose in a situation of indecisiveness. ${ }^{1}$.

A menu, in this example, is a set of employees from which Bethy has to choose whom to assign the execution of the project. For simplicity, let us assume that Alan $(A)$, Bob $(B)$, and Chris $(C)$ are the only available employees in the company, so menus are nonempty subsets of $\{A, B, C\}$. Bethy has preferences over these menus. That she prefers picking an employee from her division only rather than from the whole company means that she prefers the singleton menu $\{A\}$ (she has to choose Alan) to the grand menu $\{A, B, C\}$ (she can choose Alan, Bob, or Chris).

This preference pattern can be captured by means of the following representation. Suppose Bethy envisions two possible scenarios: the most important dimension of the project may be either finance $(f)$ or marketing $(m)$. In each scenario $s=f, m$, she is able to come up with a numerical evaluation $u_{s}:\{A, B, C\} \rightarrow \mathbb{R}$ of all employees. Namely, these evaluations are given by the following table:

\begin{tabular}{c|ccc} 
& $A$ & $B$ & $C$ \\
\hline$f$ & 5 & 8 & 6 \\
$m$ & 5 & 3 & 7
\end{tabular}

Thus, the set $U=\left\{u_{f}, u_{m}\right\}$ is a subjective state space capturing Bethy's ex ante uncertainty about her ex post preferences over candidates. Note that Alan is clearly "dominated" by Chris, no matter what subjective state eventually realizes. Hence, even though Bethy does not expect to know which dimension of the project turns out to be the most important one before she has to choose an employee, she knows ex ante that Alan is by no means the optimal employee within the grand menu. Bob and Chris, on the other hand, cannot be ranked in this manner, as which one is more valuable depends on which subjective states realizes. Hence she anticipates ex ante that the grand menu will leave her in a situation of indecisiveness between Bob and Chris. In general, when evaluating a menu, Bethy first discards all dominated employees and restricts attention to undominated ones. Thus within the grand menu $\{A, B, C\}$ she restricts attention to the submenu $\{B, C\}$ (and within the singleton menu $\{A\}$ she trivially restricts attention to $\{A\}$ itself). Moreover, being indecisiveness averse, Bethy imagines that no matter which subjective state realizes, she will always end up choosing the worst possible (undominated) employee. This yields the following, subjective state-contingent evaluation of menus:

\begin{tabular}{c|cc} 
& $\{A\}$ & $\{A, B, C\}$ \\
\hline$f$ & 5 & 6 \\
$m$ & 5 & 3
\end{tabular}

\footnotetext{
${ }^{1}$ In this sense, the decision maker who conforms to our theory prefers to avoid taking responsibility for her decisions. This interpretation was suggested to us by Klaus Nehring.
} 
Finally, Bethy obtains an ex ante numerical evaluation of a menu by aggregating the two subjective state-contingent evaluations through some increasing function from $\mathbb{R}^{2}$ to $\mathbb{R}$. If, for example, she simply sums up the two evaluations, then the singleton menu $\{A\}$ is more valuable than the grand menu $\{A, B, C\}(10>9)$.

In this paper, we take as primitive a preference relation over menus of lotteries and axiomatize the representation described above. Our model extends previous work by Guerdjikova and Zimper (2008), who model the trade-off between a desire for commitment arising from the presence of incomparable options and a desire for flexibility arising from the possibility that nature may eliminate some options between the ex ante and ex post stages. The extension is only partial since we focus on their desire for flexibility motive, thus ignoring their desire for flexibility motive. On the other hand, we axiomatize a full-fledged representation of preferences over menus whereas their main result only axiomatizes a characterization of the preference maximizing menu. Moreover, we work with a larger class of menus than they do.

Our representation exhibits a preference for commitment, once one restricts attention to undominated options. In other words, the decision maker always prefers a menu with a smaller set of undominated options. It is noteworthy that our representation does not identify which option will eventually be chosen by the decision maker (not even contingently on the subjective state since, in fact, the decision maker does not know the subjective state at the time of choosing an option). Thus our notion of indecisiveness aversion arises from the fact of having to choose without knowing one's own preferences rather than from the outcome of this choice. In this regard, our model differs from models of temptation (Gul and Pesendorfer, 2001; Dekel, Lipman, and Rustichini, 2009), regret (Sarver, 2008), costly contemplation (Ergin and Sarver, 2010), or thinking aversion (Ortoleva, 2010).

The paper is organized as follows. Section 2 presents the general setup (menus and preferences). Section 3 introduces and discusses a concept that is central to our analysis: a dominance relation over options (which is usual in the literature on preference for flexibility but has a slightly different interpretation in our model). Section 4 defines our representation concept and highlights some special cases. In Section 5 our axioms are stated and discussed. Section 6 contains our representation theorem and a proof sketch. Section 7 concludes. The Appendix contains the proof of the representation theorem and auxiliary lemmas.

\section{Setup}

Let $Z$ be a finite set of prizes and let $\Delta(Z)=\left\{\beta \in \mathbb{R}_{+}^{Z} \mid \sum_{z \in Z} \beta(z)=1\right\}$ denote the set of all probability distributions (lotteries) over $Z$ which stand for the options of our approach. As usual we may identify a prize $z \in Z$ with the lottery in $\Delta(Z)$ assigning 
probability 1 to this prize. Given $\beta, \beta^{\prime} \in \Delta(Z)$ and $\lambda \in[0,1]$, we define the $\lambda$-mixture of $\beta$ and $\beta^{\prime}$ as usual, i.e. as the lottery $\lambda \beta+(1-\lambda) \beta^{\prime} \in \Delta(Z)$ attaching to each prize $z \in Z$ the probability $\lambda \beta(z)+(1-\lambda) \beta^{\prime}(z)$. A non-empty subset $x$ of $\Delta(Z)$ is interpreted as an opportunity set or menu, i.e. as the commitment to choose some lottery $\beta \in x$ at a given later date. We refer to the choice of a menu as the ex ante stage and to the (implicit) choice of a lottery within the chosen menu as the ex post stage. We endow the set of lotteries with the Euclidean metric and the set of menus with the Hausdorff metric (see e.g. Schneider, 1993).

We restrict attention to menus that are polytopes, i.e. convex hulls of (non-empty) finite sets of lotteries. We can view these menus as determined by a finite set of linear constraints or, equivalently, we can think of the decision maker as considering finite menus but being able to randomize between options. This restriction is necessary for our representation theorem to hold (see Appendix). Let $X$ denote the set of all such menus. We consider a decision maker endowed with a weak preference relation $\succsim$ over $X$, capturing her ex ante ranking of menus. From $\succsim$ we define the strict preference relation $\succ$ and the indifference relation $\sim$ as usual, i.e. for all $x, x^{\prime} \in X, x \succ x^{\prime}$ if and only if $x \succsim x^{\prime}$ but not $x^{\prime} \succsim x$, and $x \sim x^{\prime}$ if and only if both $x \succsim x^{\prime}$ and $x^{\prime} \succsim x . \succsim$ is said to be complete if for all $x, x^{\prime} \in X$, either $x \succsim x^{\prime}$ or $x^{\prime} \succsim x$, and transitive if for all $x, x^{\prime}, x^{\prime \prime} \in X, x \succsim x^{\prime} \succsim x^{\prime \prime}$ implies $x \succsim x^{\prime \prime}$ (we will in fact assume below that $\succsim$ is both complete and transitive).

In Bethy's illustrative example, the set of prizes is the set $Z=\{A, B, C\}$ of employees, the set of options is the set $\Delta(Z)$ of lotteries over employees (any such lottery corresponding to Bethy picking an employee randomly), and the set of menus is the set $X$ of convex hulls of finite sets of lotteries over employees. Thus $X$ contains in particular the singleton menu $\{A\}$ (Bethy has to choose from her own division, i.e. pick Alan for sure) and the grand menu conv $(\{A, B, C\})$ (Bethy can choose from the whole company, i.e. pick Alan, Bob, or Chris, or randomize between them with any probabilities she wishes), where $\operatorname{conv}(\cdot)$ denotes convex hull. That she strictly prefers choosing from her own division rather than the whole company means that her preference relation $\succsim$ on $X$ is such that $\{A\} \succ \operatorname{conv}(\{A, B, C\})$.

\section{The Dominance Relation}

We first derive from $\succsim$ a dominance relation $\succsim^{*}$ over lotteries as follows: for all $\beta, \beta^{\prime} \in$ $\Delta(Z)$

$$
\beta \succsim^{*} \beta^{\prime} \Leftrightarrow\{\beta\} \sim \operatorname{conv}\left(\left\{\beta, \beta^{\prime}\right\}\right)
$$

From $\succsim^{*}$ we define the relations $\succ^{*}$ and $\sim^{*}$ as above. Moreover, since $\succsim^{*}$ can (and, in general, will) be incomplete, we denote by $\bowtie^{*}$ its noncomparability relation, i.e. for all $x, x^{\prime} \in X, x \bowtie^{*} x^{\prime}$ if and only if neither $x \succsim^{*} x^{\prime}$ nor $x^{\prime} \succsim^{*} x$. We interpret the relation 
$\beta \succsim^{*} \beta^{\prime}$ as meaning that the decision maker decisively weakly prefers $\beta$ to $\beta^{\prime}$, i.e. is certain ex ante that she will weakly prefer $\beta$ to $\beta^{\prime}$ ex post. That is to say, even if she is uncertain ex ante about her ex post preferences, all ex post preferences she deems possible are such that $\beta$ is weakly preferred to $\beta^{\prime}$. We now explain why this is naturally characterized by the singleton menu $\{\beta\}$ being indifferent (according to the primitive preference relation) to the larger (and, hence, more flexible) menu $\operatorname{conv}\left(\left\{\beta, \beta^{\prime}\right\}\right)$.

First, assume the decision maker decisively weakly prefers $\beta$ to $\beta^{\prime}$ (i.e. $\beta \succsim^{*} \beta^{\prime}$ ). Then adding $\beta^{\prime}$ to the singleton menu $\{\beta\}$ should neither improve nor worsen this menu (the convex hull is just to have a menu in $X$ ). In fact, in this case we should also have $\operatorname{conv}\left(\left\{\beta, \beta^{\prime}\right\}\right) \succsim\left\{\beta^{\prime}\right\}$, a property that will follow from our axioms (see Lemma 2 in the Appendix). Conversely, suppose that the decision maker does not decisively weakly prefer $\beta$ to $\beta^{\prime}$. This may be the case for two reasons:

- First, she may decisively strictly prefer $\beta^{\prime}$ to $\beta$ (i.e. $\beta^{\prime} \succ^{*} \beta$ ). In this case, adding $\beta^{\prime}$ to the singleton menu $\{\beta\}$ should improve this menu, so we should have $\operatorname{conv}\left(\left\{\beta, \beta^{\prime}\right\}\right) \succ\{\beta\}$ (as well as $\left\{\beta^{\prime}\right\} \sim \operatorname{conv}\left(\left\{\beta, \beta^{\prime}\right\}\right)$, see Lemma 2 in the Appendix).

- Second, she may be indecisive between $\beta$ and $\beta^{\prime}$ (i.e. $\beta \bowtie^{*} \beta^{\prime}$ ). In this case, under our assumption that she is averse to such indecisiveness, we should have $\{\beta\} \succ$ $\operatorname{conv}\left(\left\{\beta, \beta^{\prime}\right\}\right)$ and $\left\{\beta^{\prime}\right\} \succ \operatorname{conv}\left(\left\{\beta, \beta^{\prime}\right\}\right)$ (see Lemma 2 in the Appendix).

In both cases, we do not have $\{\beta\} \sim \operatorname{conv}\left(\left\{\beta, \beta^{\prime}\right\}\right)$, justifying the above definition. In Bethy's example, that Alan is dominated by Chris means that $C \succ^{*} A$, and this should be reflected in the ex ante ranking of menus by the fact that $\{C\} \sim \operatorname{conv}(\{A, C\}) \succ\{A\}$ (i.e. Bethy is indifferent between being forced to pick Chris and having to choose/randomize between Alan and Chris, and strictly prefers both of these two menus to being forced to pick Alan). The fact that Bob and Chris are not dominated by each other means that $B \bowtie^{*} C$, and this should be reflected by the fact that $\{B\} \succ \operatorname{conv}(\{B, C\})$ and $\{C\} \succ \operatorname{conv}(\{B, C\})$ (i.e. Bethy strictly prefers being forced to pick Bob, as well as being forced to pick Chris, to having to choose/randomize between Bob and Chris).

Note that our dominance relation is very similar to Kreps (1979)'s "domination" relation, and also has a similar interpretation. The only difference is that in the absence of decisive preference/dominance, the decision maker prefers larger menus in Kreps (1979)' model whereas she prefers smaller menus in our model. Of course, this just reflects the fact that Kreps (1979) assumes the decision maker expects to learn her ex post preferences before choosing a lottery whereas we assume she does not. Dekel, Lipman, and Rustichini (2001), who neither assume that the decision maker prefers larger menus nor smaller ones in the most general model they consider, also use a similar definition and interpretation in their comparative notion of subjective uncertainty. 
From $\succsim^{*}$ we define the set $c(x)$ of undominated (i.e. not strictly dominated) lotteries in a menu $x \in X$ by

$$
c(x)=\left\{\beta \in x \mid \nexists \beta^{\prime} \in x, \beta^{\prime} \succ^{*} \beta\right\} .
$$

Under our assumption that the decision maker discards all dominated lotteries and restricts attention to undominated ones, she should be indifferent between choosing a lottery in $x$ or in $c(x)$ (see Lemma 2 in the Appendix). Thus in Bethy's example, since $B \bowtie^{*} C \succ^{*} A$, we have $c(\operatorname{conv}(\{A, B, C\}))=\operatorname{conv}(\{B, C\})$ and, if Bethy restricts attention to undominated options, we should then have $\operatorname{conv}(\{A, B, C\}) \sim \operatorname{conv}(\{B, C\})$.

\section{Representation}

We look for a representation of $\succsim$ as follows:

Definition. An indecisiveness averse representation of $\succsim$ is a couple $(U, g)$, where $U \subseteq \mathbb{R}^{Z}$ is nonempty, closed, convex, and such that for all $\beta, \beta^{\prime} \in \Delta(Z)$,

$$
\beta \succsim^{*} \beta^{\prime} \Leftrightarrow\left[\forall u \in U, E u(\beta) \geq E u\left(\beta^{\prime}\right)\right]
$$

and $g: \mathbb{R}^{U} \rightarrow \mathbb{R}$ is continuous, weakly increasing on $U(X)=\left\{\left(\min _{\beta \in x} E u(\beta)\right)_{u \in U} \mid x \in X\right\}$, and such that for all $x, x^{\prime} \in X$,

$$
x \succsim x^{\prime} \Leftrightarrow g\left(\left(\min _{\beta \in c(x)} E u(\beta)\right)_{u \in U}\right) \geq g\left(\left(\min _{\beta^{\prime} \in c\left(x^{\prime}\right)} E u\left(\beta^{\prime}\right)\right)_{u \in U}\right) .
$$

The interpretation of the representation is as follows. The decision maker envisions a set of possible ex post preferences. Each of these is an expected-utility preference represented by a von Neumann-Morgenstern utility function $u \in U$, so $U$ can be interpreted as a (subjective) state space. From Equation (1), a lottery $\beta$ weakly dominates a lottery $\beta^{\prime}$ if and only if $\beta$ has weakly higher expected utility than $\beta^{\prime}$ regardless of the ex post utility function $(E(\cdot)$ denotes mathematical expectation). Equation (1) also implies that the set $c(x)$ of undominated lotteries in a menu $x$ can be computed from $U$ as:

$$
c(x)=\left\{\beta \in x \mid \nexists \beta^{\prime} \in x, E U\left(\beta^{\prime}\right)>E U(\beta)\right\}
$$

where $E U\left(\beta^{\prime}\right)>E U(\beta)$ means $E u\left(\beta^{\prime}\right) \geq E u(\beta)$ for all $u \in U$, with strict inequality for some $u \in U$. The set $c(x)$ can be shown to be nonempty and compact (see Lemma 1 in the Appendix). From Equation (2), the evaluation of a menu $x$ is fully determined by the set $c(x)$, reflecting our assumption that the decision maker restricts attention to undominated lotteries. More precisely, for each ex post utility function, the decision maker evaluates a menu $x$ by the lowest possible expected utility an undominated lottery in $x$ can give her. This reflects our assumption of aversion to indecisiveness. Finally, the different possible 
ex post utility functions are aggregated through the increasing function $g$. In Bethy's example, the subjective state space is $U=\operatorname{conv}\left(\left\{u_{f}, u_{m}\right\}\right)$, where $u_{f}, u_{m} \in \mathbb{R}^{Z}$ are the utility functions defined in the introduction, and the aggregator is $g\left(\left(r_{u}\right)_{u \in U}\right)=r_{u_{f}}+r_{u_{m}}$.

Note that the set $U$ of ex post utility functions plays a double role in the representation. First, it determines the mapping $x \rightarrow c(x)$, i.e. the set of undominated options for each menu $x$. The larger $U$, the larger $c(x)$ for a given $x$. Second, it determines the mapping $c(x) \rightarrow\left(\min _{\beta \in c(x)} E u(\beta)\right)_{u \in U}$. The larger $c(x)$, the lower $\min _{\beta \in c(x)} E u(\beta)$ for each $u \in U$ and, hence, the lower the ex ante utility of $x$ since $g$ is increasing. In the extreme case where the decision maker is not indecisive between any two lotteries (i.e. $\succsim^{*}$ is complete), $U$ can be taken to be a singleton, so the second role disappears and we have

$$
x \succsim x^{\prime} \Leftrightarrow \max _{\beta \in x} E u(\beta) \geq \max _{\beta^{\prime} \in x^{\prime}} E u\left(\beta^{\prime}\right)
$$

Thus, we are brought back to standard indirect utility for which menus are simply ranked according to their optimal options. In the opposite extreme case where the decision maker is indecisive between any two (distinct) lotteries (i.e. ${ }^{*}$ is maximally incomplete), $U$ encompasses all possible expected-utility preferences and $c(x)=x$ for all $x \in X$, so we have

$$
x \succsim x^{\prime} \Leftrightarrow g\left(\left(\min _{\beta \in x} E u(\beta)\right)_{u \in U}\right) \geq g\left(\left(\min _{\beta^{\prime} \in x^{\prime}} E u\left(\beta^{\prime}\right)\right)_{u \in U}\right) .
$$

Now, let $V=-U$ and define $h: \mathbb{R}^{V} \rightarrow \mathbb{R}$ by $h(z)=g(-z)$ for all $z \in \mathbb{R}^{V}$. Then $h$ is weakly decreasing and we have

$$
x \succsim x^{\prime} \Leftrightarrow h\left(\left(\max _{\beta \in x} E v(\beta)\right)_{v \in V}\right) \geq h\left(\left(\max _{\beta^{\prime} \in x^{\prime}} E v\left(\beta^{\prime}\right)\right)_{v \in V}\right) .
$$

This is basically Dekel, Lipman, and Rustichini (2001)'s "ordinal EU representation" with negative rather than positive monotonicity.

\section{Axioms}

In order to characterize the representation defined above, we now introduce several axioms. In order to avoid cumbersome expressions, we make use of the dominance relation $\succsim^{*}$ and the set $c(x)$ of undominated lotteries in a menu $x$ in the statement of the axioms, but one should keep in mind that all these objects are derived from the primitive preference relation $\succsim$ on $X$.

Axiom 1 (Weak order). $\succsim$ is complete and transitive.

This is a standard axiom, necessary for any utility representation.

Axiom 2 (Dominance transitivity). $\succsim^{*}$ is transitive. 
Axiom 3 (Dominance independence). For all $\beta, \beta^{\prime}, \beta^{\prime \prime} \in \Delta(Z)$ and $\lambda \in(0,1)$, if $\beta \succsim^{*} \beta^{\prime}$, then $\lambda \beta+(1-\lambda) \beta^{\prime \prime} \succsim^{*} \lambda \beta^{\prime}+(1-\lambda) \beta^{\prime \prime}$.

Axiom 4 (Dominance continuity). For all $\beta, \beta^{\prime}, \beta^{\prime \prime}, \beta^{\prime \prime \prime} \in \Delta(Z)$, the set $\{\lambda \in[0,1] \mid \lambda \beta+$ $\left.(1-\lambda) \beta^{\prime} \succsim^{*} \lambda \beta^{\prime \prime}+(1-\lambda) \beta^{\prime \prime \prime}\right\}$ is closed.

These three axioms together mean that the dominance relation $\succsim^{*}$ is a (possibly incomplete) expected-utility preference relation. In particular, Axioms 2 and 3 seem to be natural consistency properties, given our interpretation of the dominance relation as reflecting the decision maker's certain judgments about her ex post preferences. Note that transitivity of $\succsim^{*}$ neither implies nor is implied by transitivity of $\succsim$, which is imposed in Axiom 1. Independence of $\succsim^{*}$, on the other hand, is weaker than independence of $\succsim$, which is not imposed (for a detailed discussion of independence for preferences over menus, see Dekel, Lipman, and Rustichini, 2001).

Axiom 5 (Indecisiveness aversion). For all $x, x^{\prime} \in X$, if for all $\beta \in c(x)$, there exists $\beta^{\prime} \in c\left(x^{\prime}\right)$ such that $\beta \succsim^{*} \beta^{\prime}$, then $x \succsim x^{\prime}$.

This is our key axiom, stating that if every lottery that is undominated in $x$ weakly dominates some lottery that is undominated in $x^{\prime}$, then $x$ is weakly preferred to $x^{\prime}$. To understand its logic, suppose every lottery $\beta \in c(x)$ weakly dominates some lottery $\beta^{\prime} \in c\left(x^{\prime}\right)$, and let $y^{\prime}$ denote the set of all such $\beta^{\prime \prime}$ s, i.e. all lotteries in $c\left(x^{\prime}\right)$ that are weakly dominated by some lottery in $c(x)$. Since $y^{\prime}$ can basically be obtained from $c(x)$ by weakly worsening (in the sense of the dominance relation) all lotteries in $c(x)$, it seems natural for the decision maker to weakly prefer $c(x)$ to $y^{\prime}$. Now, $c\left(x^{\prime}\right)$ may also contain lotteries that do not belong to $y^{\prime}$, i.e. that are not weakly dominated by any lottery in $c(x)$. Such a lottery $\hat{\beta}^{\prime} \in c\left(x^{\prime}\right) \backslash y^{\prime}$ cannot, however, weakly dominate any lottery $\beta^{\prime} \in y^{\prime}$ (indeed, if $\hat{\beta}^{\prime} \sim^{*} \beta^{\prime}$ then $\hat{\beta}^{\prime} \in y^{\prime}$, and if $\hat{\beta}^{\prime} \succ^{*} \beta^{\prime}$ then $\beta^{\prime} \notin c\left(x^{\prime}\right)$, a contradiction in both cases), so it is necessarily noncomparable to all lotteries in $y^{\prime}$. Thus, such lotteries only create indecisiveness, so the decision maker must prefer $y^{\prime}$ to $c\left(x^{\prime}\right)$ if she is averse to indecisiveness. Moreover, if the decision maker restricts attention to undominated lotteries, then she must be indifferent between $x$ and $c(x)$, as well as between $x^{\prime}$ and $c\left(x^{\prime}\right)$. By transitivity, then, $x$ must be weakly preferred to $x^{\prime}$. Lemma 2 in the Appendix gives formal insights into this axiom.

Axiom 6 (Undominated continuity). For all $x, x^{\prime},\left(x_{n}\right)_{n \geq 1},\left(x_{n}^{\prime}\right)_{n \geq 1} \in X$ such that $c\left(x_{n}\right) \rightarrow$ $c(x)$ and $c\left(x_{n}^{\prime}\right) \rightarrow c\left(x^{\prime}\right)$, if $x_{n} \succsim x_{n}^{\prime}$ for all $n \geq 1$, then $x \succsim x^{\prime}$.

This is a continuity axiom that is distinct from Axiom 4. Since the decision maker restricts attention to undominated lotteries, the notion of continuity that is relevant to our representation involves converging sequences of sets of undominated lotteries rather than menus (note that we may have $c\left(x_{n}\right) \rightarrow c(x)$ without $x_{n} \rightarrow x$ and vice versa). 


\section{Representation Theorem}

We obtain the following result:

Theorem. There exists an indecisiveness averse representation of $\succsim$ if and only if $\succsim$ satisfies Axioms 1 to 6 .

The proof appears in the Appendix, here we only provide a brief sketch for the sufficiency part. First, by Axioms 1 to 4 , the dominance relation $\succsim^{*}$ is a (possibly incomplete) expected-utility preference relation. Hence, by Dubra, Maccheroni, and Ok (2004)'s Multi-Expected Utility Theorem, there exists a non-empty, closed, convex set $U \subseteq \mathbb{R}^{Z}$ satisfying Equation 1. This is the subjective state space. Moreover, using Axiom 5, it can be shown that $x \sim \operatorname{conv}(c(x))$ for all $x \in X$ (see Lemmas 1 and 2 in the Appendix), i.e. the decision maker restricts attention to undominated lotteries. It is therefore sufficient to establish the representation on the set $K \subseteq X$ of menus that are of the form $\operatorname{conv}(c(x))$ for some $x \in X$. On this set, $\succsim$ is complete, transitive (Axiom 1), and continuous (Axiom 6 ) and, hence, can be represented by a continuous utility function $v: K \rightarrow \mathbb{R}$. Using Axiom 5 again, it can then be shown, by means of arguments from convex analysis, that for all $x, x^{\prime} \in K$, if $\min _{\beta \in x} E u(\beta) \geq \min _{\beta^{\prime} \in x^{\prime}} E u\left(\beta^{\prime}\right)$ for all $u \in U$ then $x \succsim x^{\prime}$. Consequently, one can define a continuous and increasing aggregator $g: \mathbb{R}^{U} \rightarrow \mathbb{R}$ such that $v(x)=g\left(\left(\min _{\beta \in x} E u(\beta)\right)_{u \in U}\right)$ for all $x \in K$, which establishes Equation 2 and completes the proof of the sufficiency part.

\section{Conclusion}

We have introduced and axiomatized a representation of preferences over menus capturing the notion of indecisiveness aversion. In our representation, the decision maker's ex ante uncertainty about her ex post preferences is captured by means of a subjective state space. Since this uncertainty does not resolve before the choice of option, it gives rise to indecisiveness at the ex post stage. More specifically, the decision maker discards options that are clearly dominated, and evaluates the remaining set of undominated options pessimistically, as if she would end up with the worst possible undominated option, no matter which subjective state realizes. This gives rise to a preference for commitment, in the sense of preferring menus with fewer undominated options.

Our representation is ordinal in the sense that our aggregator is only required to be weakly increasing and continuous. A natural refinement of the present model would be to look for a more specific representation in which the aggregator has a linear form. That is to say, one could look for a positive measure $\mu$ on $U$ such that, for all $x, x^{\prime} \in X$,

$$
x \succsim x^{\prime} \Leftrightarrow \int_{u \in U}\left(\min _{\beta \in c(x)} E u(\beta)\right) d \mu(u) \geq \int_{u \in U}\left(\min _{\beta^{\prime} \in c\left(x^{\prime}\right)} E u\left(\beta^{\prime}\right)\right) d \mu(u) .
$$


One thing to note about this representation is that it is not truly linear. This is because it is not true that $c\left(\lambda x+(1-\lambda) x^{\prime}\right)=\lambda c(x)+(1-\lambda) c\left(x^{\prime}\right)$ in general. In fact, it is only true that $c\left(\lambda x+(1-\lambda) x^{\prime}\right) \subseteq \lambda c(x)+(1-\lambda) c\left(x^{\prime}\right)$ but the converse does not hold because, roughly speaking, by mixing between two menus one can get rid of some undominated options. Therefore, this representation does not imply the independence axiom, but only the following, weaker axiom: For all $x_{1}, x_{2}, \bar{x}, y_{1}, y_{2} \in X$ and $\lambda \in(0,1)$ such that $\operatorname{conv}\left(c\left(y_{i}\right)\right)=\operatorname{conv}\left(\lambda c\left(x_{i}\right)+(1-\lambda) c(\bar{x})\right), i=1,2$, if $x_{1} \succsim x_{2}$ then $y_{1} \succsim y_{2}$. This makes it tempting to try to work on the class $\{\operatorname{conv}(c(x)) \mid x \in X\}$ and parallel the proof of Dekel, Lipman, and Rustichini (2001); Dekel, Lipman, Rustichini, and Sarver (2007a)'s additive representation theorem. However, since this class is not convex, a similar argument to theirs (in particular for Lemma S11 in Dekel, Lipman, Rustichini, and Sarver, 2007b) is not at hand in our model. We leave the problem of axiomatizing a linear representation of indecisiveness averse preferences open for future research.

\section{Appendix}

Lemma 1. Assume there exists $U \subseteq \mathbb{R}^{Z}$ such that ${ }^{*}$ satisfies Equation (1). Then for all $x \in X, \operatorname{conv}(c(x)) \in X$.

Proof. First, $c(x)$ is nonempty since $x$ is compact (Eliaz and Ok, 2006, Lemma 3). Since a polytope has only finitely many faces and each of these faces is closed, it is sufficient to show that $c(x)$ is a union of faces of $x$. Let $\beta \in c(x)$. We know that $\beta$ belongs to the relative interior of some face $f$ of $x$ (Rockafellar, 1970, Theorem 18.2). It is sufficient to show that $f \subseteq c(x)$. Suppose there exists $\beta^{\prime} \in f$ such that $\beta^{\prime} \notin c(x)$. Then, clearly, $\beta^{\prime} \neq \beta$. Moreover, by Equation 1 and the definition of $c(x)$, there exists $\bar{\beta}^{\prime} \in x$ such that $U \cdot\left(\bar{\beta}^{\prime}-\beta^{\prime}\right)>0$, where $\cdot$ denotes scalar product and $U \cdot\left(\bar{\beta}^{\prime}-\beta^{\prime}\right)>0$ means $u \cdot\left(\bar{\beta}^{\prime}-\beta^{\prime}\right) \geq 0$ for all $u \in U$ with strict inequality for at least some $u \in U$. Now, since $\beta$ belongs to the relative interior of $f$, there exists $\beta^{\prime \prime} \in f$ and $\lambda \in(0,1)$ such that $\beta=\lambda \beta^{\prime}+(1-\lambda) \beta^{\prime \prime}$ (Rockafellar, 1970, Theorem 6.4). Let $\bar{\beta}=\lambda \bar{\beta}^{\prime}+(1-\lambda) \beta^{\prime \prime}$. Then $U \cdot(\bar{\beta}-\beta)=\lambda U \cdot\left(\bar{\beta}^{\prime}-\beta^{\prime}\right)>0$, so $\beta \notin c(x)$, a contradiction.

Lemma 2. Assume $\succsim$ satisfies Axioms 1 to 5. Then:

1. For all $\beta, \beta^{\prime}, \beta^{\prime \prime} \in \Delta(Z)$ and $\lambda \in(0,1), \beta \succsim^{*} \beta^{\prime}$ if and only if $\lambda \beta+(1-\lambda) \beta^{\prime \prime} \succsim^{*}$ $\lambda \beta^{\prime}+(1-\lambda) \beta^{\prime \prime}$.

2. For all $x, x^{\prime} \in X$, if $c(x) \subseteq c\left(x^{\prime}\right)$, then $x \succsim x^{\prime}$.

3. For all $x \in X, \operatorname{conv}(c(x)) \in X$.

4. For all $x \in X, x \sim \operatorname{conv}(c(x))$. 
5. For all $\beta, \beta^{\prime} \in \Delta(Z)$,

$$
\begin{aligned}
& \beta \sim^{*} \beta^{\prime} \Leftrightarrow\{\beta\} \sim \operatorname{conv}\left(\left\{\beta, \beta^{\prime}\right\}\right) \sim\left\{\beta^{\prime}\right\}, \\
& \beta \succ^{*} \beta^{\prime} \Leftrightarrow\{\beta\} \sim \operatorname{conv}\left(\left\{\beta, \beta^{\prime}\right\}\right) \succ\left\{\beta^{\prime}\right\}, \\
& \beta \bowtie^{*} \beta^{\prime} \Leftrightarrow\left[\{\beta\} \succ \operatorname{conv}\left(\left\{\beta, \beta^{\prime}\right\}\right) \text { and }\left\{\beta^{\prime}\right\} \succ \operatorname{conv}\left(\left\{\beta, \beta^{\prime}\right\}\right)\right] .
\end{aligned}
$$

Proof. 1. Follows from Axioms 1 to 4 (Dubra, Maccheroni, and Ok, 2004, Lemma 1).

2. Follows immediately from Axiom 5.

3. By Axioms 1 to 4, there exists a nonempty, closed, convex set $U \subseteq \mathbb{R}^{Z}$ such that ${ }^{*}$ satisfies (1) (Dubra, Maccheroni, and Ok, 2004). Hence the result follows from Lemma 1.

4. By parts 2 and 3 of this lemma, it is sufficient to prove that $c(\operatorname{conv}(c(x)))=c(x)$. First, we show that for all $\beta \in x$, there exists $\beta^{\prime} \in c(x)$ such that $\beta^{\prime} \succsim^{*} \beta$. Let $y=\{\bar{\beta} \in$ $x \mid \bar{\beta} \succsim^{*} \beta$. Since $x$ is compact and $\succsim^{*}$ is continuous (Dubra, Maccheroni, and Ok, 2004, Proposition 1), $y$ is compact and, hence, there exists $\beta^{\prime} \in y$ such that $\bar{\beta} \succ^{*} \beta^{\prime}$ for no $\bar{\beta} \in y$ (Eliaz and Ok, 2006, Lemma 3). Suppose $\bar{\beta} \succ^{*} \beta^{\prime}$ for some $\bar{\beta} \in x \backslash y$. Since $\beta^{\prime} \succsim^{*} \beta$ by definition of $y$, it follows that $\bar{\beta} \succ^{*} \beta$ by transitivity of $\succsim^{*}$, so $\bar{\beta} \in y$, a contradiction. Hence $\beta^{\prime} \in c(x)$.

Now, by definition, $c(x)=\left\{\beta \in x \mid \nexists \beta^{\prime} \in x, \beta^{\prime} \succ^{*} \beta\right\}$ and $c(\operatorname{conv}(c(x)))=\{\beta \in$ $\left.\operatorname{conv}(c(x)) \mid \nexists \beta^{\prime} \in \operatorname{conv}(c(x)), \beta^{\prime} \succ^{*} \beta\right\}$. Let $y^{\prime}=\left\{\beta \in \operatorname{conv}(c(x)) \mid \nexists \beta^{\prime} \in x, \beta^{\prime} \succ^{*} \beta\right\}$. Then $y^{\prime}=c(x) \cap \operatorname{conv}(c(x))=c(x)$. We show that $c(\operatorname{conv}(c(x)))=y^{\prime}$. Clearly, $y^{\prime} \subseteq$ $c(\operatorname{conv}(c(x)))$ since $\operatorname{conv}(c(x)) \subseteq x$. Conversely, let $\beta \in \operatorname{conv}(c(x)) \backslash y^{\prime}$. Then there exists $\beta^{\prime} \in x$ such that $\beta^{\prime} \succ^{*} \beta$. By the argument above, there then exists $\beta^{\prime \prime} \in c(x)$ such that $\beta^{\prime \prime} \succsim^{*} \beta^{\prime}$ and, hence, $\beta^{\prime \prime} \succ^{*} \beta$, so $\beta \notin c(\operatorname{conv}(c(x)))$. Hence $c(\operatorname{conv}(c(x))) \subseteq y^{\prime}$.

5. The indifference property follows immediately from the definition of $\succsim^{*}$. Now, for all $\beta, \beta^{\prime} \in \Delta(Z)$, we obviously have $c(\{\beta\})=\{\beta\}$ and $c\left(\left\{\beta^{\prime}\right\}\right)=\left\{\beta^{\prime}\right\}$. Moreover, by part 1 of this lemma,

$$
c\left(\operatorname{conv}\left(\left\{\beta, \beta^{\prime}\right\}\right)\right)= \begin{cases}\{\beta\} & \text { if } \beta \succ^{*} \beta^{\prime}, \\ \left\{\beta^{\prime}\right\} & \text { if } \beta^{\prime} \succ^{*} \beta, \\ \left.\operatorname{conv}\left(\left\{\beta, \beta^{\prime}\right\}\right)\right) & \text { if } \beta \sim^{*} \beta^{\prime} \text { or } \beta \bowtie^{*} \beta^{\prime} .\end{cases}
$$

We now show that $\beta \succsim^{*} \beta^{\prime}$ implies $\operatorname{conv}\left(\left\{\beta, \beta^{\prime}\right\}\right) \succsim\left\{\beta^{\prime}\right\}$. Suppose $\beta \succsim^{*} \beta^{\prime}$ and $\left\{\beta^{\prime}\right\} \succ \operatorname{conv}\left(\left\{\beta, \beta^{\prime}\right\}\right)$. Then $\{\beta\} \sim \operatorname{conv}\left(\left\{\beta, \beta^{\prime}\right\}\right)$ by definition of $\succsim^{*}$ and, hence, $\left\{\beta^{\prime}\right\} \succ$ $\{\beta\}$ by transitivity of $\succsim$. But since $c(\{\beta\})=\{\beta\}, c\left(\left\{\beta^{\prime}\right\}\right)=\left\{\beta^{\prime}\right\}$, and $\beta \succsim^{*} \beta^{\prime}$, we have $\{\beta\} \succsim\left\{\beta^{\prime}\right\}$ by Axiom 5, a contradiction. This establishes the strict preference property as well as the $\Leftarrow$ part of the noncomparability property. For the $\Rightarrow$ part, assume $\beta \bowtie^{*} \beta^{\prime}$. Then $c(\{\beta\}) \subseteq c\left(\operatorname{conv}\left(\left\{\beta, \beta^{\prime}\right\}\right)\right)$ and $c\left(\left\{\beta^{\prime}\right\}\right) \subseteq c\left(\operatorname{conv}\left(\left\{\beta, \beta^{\prime}\right\}\right)\right)$, so we have $\{\beta\} \succsim \operatorname{conv}\left(\left\{\beta, \beta^{\prime}\right\}\right)$ and $\left\{\beta^{\prime}\right\} \succsim \operatorname{conv}\left(\left\{\beta, \beta^{\prime}\right\}\right)$ by part 2 of this lemma. Suppose these two preferences are in fact indifferences. Then $\beta \sim^{*} \beta^{\prime}$, a contradiction. Hence one of 
the two must be strict. Suppose the other one is an indifference. Then we face the same contradiction as above. Hence both preferences are strict.

Proof of the Representation Theorem. Obviously, Axiom 1 is necessary for a representation to exist. Given this axiom, we know that $\succsim^{*}$ is reflexive and, hence, Axioms 2 to 4 are necessary and sufficient for the existence of a nonempty, closed, convex set $U \subseteq \mathbb{R}^{Z}$ such that ${ }^{*}$ satisfies Equation (1) (Dubra, Maccheroni, and Ok, 2004). It remains to prove that Axioms 5 and 6 are necessary and sufficient for the existence of a continuous and weakly increasing aggregator $g: U(X) \rightarrow \mathbb{R}$ such that $\succsim$ satisfies Equation (2). It is easy to check that these axioms are necessary. The remainder of this proof is devoted to the sufficiency part.

Assume $\succsim$ satisfies Axioms 1 to 6 . Let $K=\{\operatorname{conv}(c(x)) \mid x \in X\}$. Clearly, for all $x \in X$ and $u \in U$, we have $\min _{\beta \in c(x)} E u(\beta)=\min _{\beta \in \operatorname{conv}(c(x))} E u(\beta)$. Hence, by parts 3 and 4 of Lemma 2 , it is sufficient to find a continuous and weakly increasing aggregator $g$ such that, for all $x, x^{\prime} \in K$,

$$
x \succsim x^{\prime} \Leftrightarrow g\left(\left(\min _{\beta \in x} E u(\beta)\right)_{u \in U}\right) \geq g\left(\left(\min _{\beta^{\prime} \in x^{\prime}} E u\left(\beta^{\prime}\right)\right)_{u \in U}\right) .
$$

Since $K$ is a subset of a separable metric space (Klein and Thompson, 1984), Axioms 1 and 6 imply the existence of a continuous utility function $v: K \rightarrow \mathbb{R}$ such that, for all $x, x^{\prime} \in$ $K, x \succsim x^{\prime}$ if and only if $v(x) \geq v\left(x^{\prime}\right)$ (Debreu, 1954). We now claim that for all $x, x^{\prime} \in K$, if $\min _{\beta \in x} E u(\beta) \geq \min _{\beta^{\prime} \in x^{\prime}} E u\left(\beta^{\prime}\right)$ for all $u \in U$, then $x \succsim x^{\prime}$. If the claim is correct, then we can define the aggregator $g: U(K)=U(X) \rightarrow \mathbb{R}$ by, for all $\left(r_{u}\right)_{u \in U} \in U(K)$, $g\left(\left(r_{u}\right)_{u \in U}\right)=v(x)$ for any $x \in K$ such that $\left(\min _{\beta \in x} E u(\beta)\right)_{u \in U}=\left(r_{u}\right)_{u \in U}$. Moreover, it is clear that $g$ is then weakly increasing, so the proof is complete.

To prove the claim, let $x \in K$ and define the sets

$$
\begin{aligned}
& y=\left\{\gamma \in \mathbb{R}^{Z} \mid \forall u \in U, u \cdot \gamma \geq \min _{\beta \in x} u \cdot \beta\right\}, \\
& y^{\prime}=\left\{\gamma \in \mathbb{R}^{Z} \mid \exists \beta \in x, U \cdot \gamma \geq U \cdot \beta\right\} .
\end{aligned}
$$

By Equation (1) and Axiom 5, it is sufficient to show that $y^{\prime}=y$. Define the set $k=$ $\left\{\gamma \in \mathbb{R}^{Z} \mid U \cdot \gamma \geq 0\right\}$. Then $k$ is a closed convex cone and, more precisely, is the polar of the cone generated by $-U$. Clearly, $y^{\prime}=x+k$. Since $x$ is a polytope and $k$ is closed and convex, $y^{\prime}$ is closed and convex (Rockafellar, 1970, Theorem 20.3) and, hence, is equal to the intersection of all closed half-spaces containing it (Rockafellar, 1970, Theorem 11.5). For all $u \in \mathbb{R}^{Z}$, define the set $h_{u}=\left\{\gamma \in \mathbb{R}^{Z} \mid u \cdot \gamma \geq \inf _{\gamma^{\prime} \in y^{\prime}} u \cdot \gamma^{\prime}\right\}$. Clearly, we have $y^{\prime}=\bigcap\left\{h_{u} \mid u \in \mathbb{R}^{Z}\right\}=\bigcap\left\{h_{u} \mid u \in V\right\}$, where $V=\left\{u \in \mathbb{R}^{Z} \mid \inf _{\gamma^{\prime} \in y^{\prime}} u \cdot \gamma^{\prime}>-\infty\right\}$. By definition, $-V$ is the barrier cone of $y^{\prime}$ and, hence, is the polar of the recession cone of $y^{\prime}$ (Rockafellar, 1970, Corollary 14.2.1). But since $x$ is a polytope, the recession cone of 
$y^{\prime}$ is the recession cone of $k$ and, since $k$ is a cone, the recession cone of $k$ is $k$. Thus $-V$ is the polar of $k$ and, hence is the cone generated by $-U$. Since $U$ is convex, this latter cone is $\bigcup\{\lambda U \mid \lambda \geq 0\}$ and, since $h_{u}=h_{\lambda u}$ for all $\lambda>0$ by definition, we have $y^{\prime}=\bigcap\left\{h_{u} \mid u \in U\right\}$. Finally, since $k$ is a cone and $x$ is a polytope, $\inf _{\gamma^{\prime} \in y^{\prime}} u \cdot \gamma^{\prime}>-\infty$ implies $\inf _{\gamma^{\prime} \in y^{\prime}} u \cdot \gamma^{\prime}=\min _{\gamma^{\prime} \in x} u \cdot \gamma^{\prime}$, so the latter equality implies $y^{\prime}=y$.

\section{References}

DeBreu, G. (1954): "Representation of a preference ordering by a numerical function," in Decision Processes, ed. by R. Thrall, C. Coombs, and R. Davis. John Wiley.

Dekel, E., B. L. Lipman, and A. Rustichini (2001): "Representing preferences with a unique subjective state space," Econometrica, 69(4), 891-934.

(2009): "Temptation-driven preferences," Review of Economic Studies, 76(3), 937-971.

Dekel, E., B. L. Lipman, A. Rustichini, and T. Sarver (2007a): "Representing preferences with a unique subjective state space: A corrigendum," Econometrica, 75, $591-600$.

- (2007b): "Supplement to "Representing preferences with a unique subjective state space: Corrigendum"," Econometrica Supplementary Material, 75.

Dubra, J., F. Maccheroni, and E. A. OK (2004): "Expected utility theory without the completeness axiom," Journal of Economic Theory, 115(1), 118-133.

Eliaz, K., AND E. A. OK (2006): "Indifference or indecisiveness? Choice-theoretic foundations of incomplete preferences," Games and Economix Behavior, 56(1), 61-86.

Epstein, L., M. Marinacci, And K. Seo (2007): "Coarse contingencies and ambiguity," Theoretical Economics, 2, 355-394.

Ergin, H., And T. SARver (2010): "A unique costly contemplation representation," Econometrica, 78(4), 1285-1339.

Guerdjikova, A., AND A. Zimper (2008): "Flexibility of choice versus reduction of ambiguity," Social Choice and Welfare, 30, 507-526.

Gul, F., And W. Pesendorfer (2001): "Temptation and self-control," Econometrica, 69(6), 1403-1435.

Klein, E., And A. C. Thompson (1984): Theory of correspondences: Including applications to mathematical economics. Wiley and Sons. 
KREPS, D. M. (1979): "A representation theorem for "preference for flexibility"," Econometrica, 47(3), 565-577.

Nehring, K. (1999): "Preference for flexibility in a Savage framework," Econometrica, 67, 101-119.

Ortoleva, P. (2010): "The price of flexibility: Towards a theory of thinking aversion,"

OzDEnOREn, E. (2002): "Completing the state space with subjective states," Journal of Economic Theory, 105, 531-539.

Rockafellar, R. T. (1970): Convex analysis. Princeton University Press.

SARVER, T. (2008): "Anticipating regret: why fewer options may be better," Econometrica, 76, 263-305.

SCHneIDeR, R. (1993): Convex bodies: the Brunn-Minkowski theory. Canbridge University Press. 